\title{
Analysis of anatomical variations of the lingular artery of the left upper lobe using 3D computed tomography angiography and bronchography
}

\author{
Hao He^, Peng Chen, Xiaofeng Chen, Pei Yuan Wang, Shuo Yan Liu, Feng Wang \\ Department of Thoracic Surgery, Fujian Medical University Cancer Hospital, Fujian Cancer Hospital, Fuzhou, China \\ Contributions: (I) Conception and design: H He; (II) Administrative support: F Wang, SY Liu; (III) Provision of study materials or patients: All \\ authors; (IV) Collection and assembly of data: H He; (V) Data analysis and interpretation: H He; (VI) Manuscript writing: All authors; (VII) Final \\ approval of manuscript: All authors. \\ Correspondence to: Feng Wang. Department of Thoracic Surgery, Fujian Medical University Cancer Hospital, Fujian Cancer Hospital, Fuzhou \\ 350014, China. Email: swfmd120@163.com.
}

Background: Thoracic surgeons must be familiar with the anatomy of the pulmonary artery during
segmentectomy and segmentectomy. But pulmonary arteries have numerous variations and aberrant
branching patterns. The purpose of the present study was to analyze the anatomical variations and
frequencies of the lingular artery of the left upper lobe (LUL) using 3D computed tomography angiography
and bronchography (3D-CTAB). Methods: We retrospectively studied 166 patients having undergone lobectomy or segmentectomy from January to December 2020 at Fujian Medical University Cancer Hospital's Department of Thoracic Surgery. All patients underwent 3D reconstruction using 3D-CTAB before surgery.

Results: The lingular segment was supplied by 1 artery in $45.18 \%$ of cases, 2 arteries in $46.39 \%$ of cases, and 3 arteries in $8.43 \%$ of cases. The branching patterns of the lingular artery included $119(71.68 \%)$ cases with interlobar origin, 35 (21.08\%) cases with interlobar and mediastinal origin, and 13 (7.83\%) cases with mediastinal origin. The interlobar lingular artery include superior lingular artery (A4) and inferior lingular artery (A5). The interlobar lingular artery type was A4a, A4b, A5 in 7.23\% of cases; A4 and A4b+5 in 3.01\% of cases; and $\mathrm{A} 4 \mathrm{~b}$ and $\mathrm{A} 4 \mathrm{a}+5$ in $4.82 \%$ of cases. The mediastinal lingular artery was divided into the following 5 types: 'A4', 'A4b', 'A4b+5', 'A4b+5a', and 'A4+5'. The most common type was A4 (12.05\%, 20/166) in 166 patients. The interlobar lingular artery had the following 5 patterns of variation: 'A4+5', 'A4, A5', 'A4a, A4b, $\mathrm{A} 5$ ', 'A4a, A4b+5', and 'A4b, A4a+5'. The single interlobar lingular artery (A4+5) was the most common type in $38.55 \%$ of cases. In $24.10 \%$ of cases, A5 came from A8 or A8+9. Besides In $8.43 \%$ of cases, the origin of A5 was close to A8 or A8+9.

Conclusions: We identified the left various lingular artery branching patterns with 3D-CTAB in patients and defined the frequency of anatomic variations. $3 \mathrm{D}-\mathrm{CTAB}$ is useful for finding these variations.

Keywords: Left upper lobe (LUL); 3D computed tomography angiography and bronchography (3D-CTAB); lingular artery; anatomy; segmentectomy

Submitted May 18, 2021. Accepted for publication Aug 11, 2021.

doi: $10.21037 /$ jtd-21-1141

View this article at: https://dx.doi.org/10.21037/jtd-21-1141

^ ORCID: 0000-0001-9738-6015. 


\section{Introduction}

Because of the widespread use of lung computed tomography (CT) increasing numbers of small pulmonary nodules are detected. Some previously published studies have shown that, for small pulmonary nodules, the prognosis of segmentectomy is no worse than that of lobectomy (1-4). Anatomic precise segmentectomy can be used as a treatment option for benign nodules or early lung cancer $(5,6)$.

Anatomic variations of the pulmonary artery make pulmonary segmentectomy more difficult than standard lobectomy. Therefore, surgeons must have a comprehensive and accurate understanding of the models of pulmonary artery, including rare anatomical variations. This is important for the safe and accurate completion of pulmonary segmentectomy. However, without a good understanding of the artery anatomical model and the various variations, it is difficult to understand pulmonary artery anatomy. Traditional methods, such as autopsy $(7,8)$ and 2D computed tomography (2DCT) (9) have been used to understand the anatomy of the lung. The anatomical structure of the lung was analyzed by some scholars in the 1940 s and 1950s using cadavers $(7,8)$. However, this has some shortcomings, such as complex operation, the low number of cadavers, and abnormal physiological state of the lungs of cadavers. 2DCT cannot truly reproduce the 3D structure of lung anatomy (10).

With the development of medical technology, 3D reconstruction of the anatomical structure of the pulmonary artery, based on preoperative lung CT data, can be a good guide for surgery (11). The physiological state of pulmonary vessels and bronchi can be observed by 3D CT angiography and bronchography (3D-CTAB).

However, the anatomic variations of the artery are still controversial, particularly with respect to branching of the lingular artery $(7,8,12-15)$. There have been few studies on the left upper lobe (LUL) pulmonary lingular artery model.

The purpose of the present study was to describe variations and frequencies in the LUL pulmonary lingular artery model using CT angiography and 3D reconstruction so as to assist thoracic surgeons with LUL segmentectomies. We present the following article in accordance with the STROBE reporting checklist (available at https://dx.doi. org/10.21037/jtd-21-1141).

\section{Methods}

A total of 166 patients with left pulmonary lesions, and with no history of left pulmonary resection, were included in the present study from January to December 2020. The study was undertaken at the Department of Thoracic Surgery, Fujian Medical University Cancer Hospital, Fujian Cancer Hospital. All patients underwent 3D reconstruction before surgery, including segmentectomy and subsegmentectomy (Figure 1).

\section{Statistical analysis}

All statistical analyses were conducted using SPSS Version 22 software (SPSS, Inc., Chicago, IL, USA).

\section{D-CTAB}

We analyzed the bronchial patterns on 3D-CTAB imaging using 256 channel MDCT (Multi-detector Computed Tomography General Electric Healthcare, Boston, MA, USA). A total of $35 \mathrm{~mL}$ contrast medium was given intravenously at a rate of $5 \mathrm{~mL} / \mathrm{s}$, followed immediately by $20 \mathrm{~mL}$ normal saline intravenously. Slice thickness was $0.625 \mathrm{~mm}$. A 3D image was then constructed from $0.625-\mathrm{mm}$ slices; data were obtained by contrast-enhanced CT images using 3D image reconstruction software (EDDA Technology, Princeton, New Jersey, USA).

\section{Nomenclature of the pulmonary artery}

We used the same nomenclature to name the segmental or subsegmental bronchi as Yamashita and Hiroaki Nomori as follows: the lingular artery consists of the superior lingular artery (A4) and inferior lingular artery (A5). A4 is the superior lingular artery, which is divided into the lateral artery (A4a) and the medial artery (A4b). A5 is the inferior lingular artery, which is divided into the superior artery (A5a) and the inferior artery (A5b) $(12,16)$.

In our study a thoracic surgeon and radiologist identified the branching lingular artery and the segments and subsegments according to the lumen diameter of the lingular artery and the branching direction of the 3D lingular artery. If there were difficult cases, other radiologist and surgeons were consulted to improve accuracy.

\section{Ethical statement}

The study was conducted in accordance with the Declaration of Helsinki (as revised in 2013). The study was approved by ethics board of Fujian Medical University 


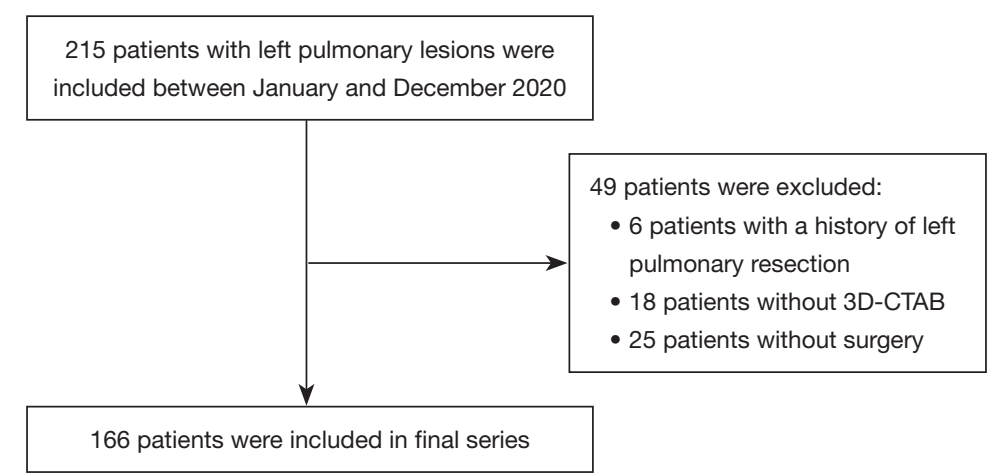

Figure 1 Study flowchart. 3D-CTAB, 3D computed tomography angiography and bronchography.

Table 1 Number of lingular arteries

\begin{tabular}{|c|c|c|c|c|}
\hline & $\begin{array}{l}\text { Present study } \\
\quad(n=166), \%\end{array}$ & $\begin{array}{l}\text { Cory and Valentine } \\
(n=107)(13), \%\end{array}$ & $\begin{array}{l}\text { Fourdrain et al. } \\
(n=44)(14), \%\end{array}$ & $\begin{array}{c}\text { Dejima et al. } \\
(n=199)(15), \%\end{array}$ \\
\hline \multicolumn{5}{|l|}{ No. lingular arteries } \\
\hline 1 & 45.18 & 89.70 & 77.30 & 55.30 \\
\hline 2 & 46.39 & 6.50 & 22.70 & 44.70 \\
\hline $\begin{array}{l}\text { Common trunk: interlobar upper division } \\
\text { artery-interlobar lingular artery }\end{array}$ & 10.89 & 17.80 & 20.50 & - \\
\hline
\end{tabular}

Table 2 Lingular artery branching patterns

\begin{tabular}{|c|c|c|c|c|c|c|}
\hline $\begin{array}{l}\text { Lingular artery branching } \\
\text { patterns }\end{array}$ & $\begin{array}{l}\text { Present study } \\
(n=166), \%\end{array}$ & $\begin{array}{c}\text { Jiang } \\
(\mathrm{n}=85)(8), \%\end{array}$ & $\begin{array}{c}\text { Yamashita } \\
(n=170)(16), \%\end{array}$ & $\begin{array}{l}\text { Nagata et al. } \\
(n=186)(17), \%\end{array}$ & $\begin{array}{c}\text { Dejima et al. } \\
(n=199)(15), \%\end{array}$ & $\begin{array}{c}\text { Boyden and } \\
\text { Hartmann }(n=50)(7), \%\end{array}$ \\
\hline Interlobar type & 71.68 & 76.00 & 72.90 & 63.90 & 70.85 & 74.00 \\
\hline Mediastinal type & 7.83 & 6.00 & 9.70 & 9.20 & 10.05 & 8.00 \\
\hline
\end{tabular}

Cancer Hospital, Fujian Cancer Hospital (NO.: 2017-050$01)$ and individual consent for this retrospective analysis was waived.

\section{Results}

A total of 166 patients (72 males and 94 females), were enrolled in the present study from January to December 2020. The median age was 57 years (range, $25-85$ years). All patients underwent segmentectomy or subsegmentectomy after $3 \mathrm{D}$ reconstruction.

The lingular segment was supplied by 1 artery in $45.18 \%$ of cases, 2 arteries in $46.39 \%$ of cases, and 3 arteries in
$8.43 \%$ of cases. Interlobarupper division arteries (A1-3) and interlobar lingular arteries were common trunk in $10.89 \%$ of cases, A3a originating from the lingular artery accounted for $5 \%$ and $\mathrm{A} 1+2 \mathrm{c} 3.06 \%$ (Table 1 ).

In the present study, we found that the branching patterns of the lingular artery included $119(71.68 \%)$ cases with interlobar origin, 35 (21.08\%) with interlobar and mediastinal origin, and 13 (7.83\%) with mediastinal origin (Table 2).

The mediastinal lingular artery was found in $28.91 \%$ of patients, and was divided into the following 5 types: the mediastinal lingular artery A4b was found in $4.21 \%$ cases, $\mathrm{A} 4$ was found in $12.05 \%$ cases, $\mathrm{A} 4 \mathrm{~b}+5$ was found in $3.61 \%$ 
Table 3 Branching of the lingular artery of the left upper lobe

\begin{tabular}{|c|c|c|c|c|}
\hline & $\begin{array}{l}\text { Present study } \\
\text { (n=166), \% }\end{array}$ & $\begin{array}{c}\text { Jiang } \\
(\mathrm{n}=85)(8), \%\end{array}$ & $\begin{array}{c}\text { Nomori and } \\
\text { Okada (12), \% }\end{array}$ & $\begin{array}{l}\text { Boyden and Hartmann } \\
\qquad(n=50)(7), \%\end{array}$ \\
\hline Mediastinal lingular artery & 28.91 & 24.00 & - & 30.00 \\
\hline $\mathrm{A} 4 \mathrm{~b}$ & 4.21 & 12.00 & - & 14.00 \\
\hline A4 & 12.05 & 6.00 & - & 6.00 \\
\hline $\mathrm{A} 4+5$ & 7.83 & 6.00 & 18.00 & 8.00 \\
\hline A5 & - & - & - & 2.00 \\
\hline Interlobar lingular artery & 71.68 & 76.00 & 70.00 & 74.00 \\
\hline$A 4 a, A 4 b+5$ & 3.01 & - & - & - \\
\hline $\mathrm{A} 4 \mathrm{~b}, \mathrm{~A} 4 \mathrm{a}+5$ & 4.82 & - & - & - \\
\hline
\end{tabular}

cases, A4b+5a was found in $1.20 \%$ cases, and A4+5 was found in $7.83 \%$ cases. All of the mediastinal lingular artery originated from the pulmonary artery in common with A3. The mediastinal lingular arteries were all deep in the vein of the anterior and posterior segments of the LUL. The interlobar lingular artery was found in $71.68 \%$ of patients, with the following 5 patterns of variation: single interlobar artery $(\mathrm{A} 4+5)$ in $38.55 \%$ cases, 2 interlobar arteries separately (A4 and A5) in 17.47\% cases, 3 interlobar arteries separately (A4a, A4b, A5) in 7.23\% cases, A4b and A5 stem both arising from the pulmonary artery (A4a and $A 4 b+5)$ in $3.01 \%$ cases, and $\mathrm{A} 4 \mathrm{a}$ and $\mathrm{A} 5$ stem both arising from the pulmonary artery (A4b and $\mathrm{A} 4 \mathrm{a}+5)$ in $4.82 \%$ cases (Table 3). In $24.10 \%$ of cases, A5 came from A8 or A8+9. Besides, in $8.43 \%$ of cases, the origin of A5 was close to A8 or A8+9.

\section{Discussion}

Thoracic thin-section CT has led to an improved detection rate of small lung lesions. Some previously published studies have shown that, for metastatic lung tumors, benign nodules, or early lung cancer, the prognosis of segmentectomy is no worse than that of lobectomy (1-4,18-21). The JCOG0802 study, which compared segmentectomy and lobectomy in patients with invasive peripheral nonsmall cell lung cancer tumors with a maximum diameter $\leq 2 \mathrm{~cm}$, showed that segmentectomy had a higher rate of air leakage $(6.5 \%$ vs. $3.8 \%, \mathrm{P}=0.04)$, but there was no difference in other complication rates (5). The JCOG0804 study evaluated the efficacy and safety of sublobar resection for ground-glass opacity-dominant peripheral lung cancer with a maximum tumor diameter $\leq 2 \mathrm{~cm}$ and consolidation tumor ratio $\leq 0.25$ (6). The findings of the JCOG0804 study indicated that sublobar resection should be the preferred surgical procedure. In addition, pulmonary segmentectomy is a surgical option for patients who were unconditionally undergoing standard lobectomy (22).

Because of the anatomic variations of the artery, thoracic surgeons must be familiar with artery anatomy and its variations in order to successfully perform segmental pulmonary resection. It is well known that the most common variation is a mediastinal lingular artery $(16,23,24)$. However, further specific classification and occurrence probability are rarely mentioned in previously published reports.

In the present study, based on 3D-CTAB reconstruction, the segmental and subsegmental lingular arteries of the LUL were studied and classified in detail. The present study is a systematic radiological analysis of the lingular artery of the LUL following 3D-CTAB reconstruction, and highlights the differences between our results and those of previous reports. The pulmonary parenchyma and pulmonary bronchus significantly interfered with the 
anatomical analysis of the arterial variation pattern. However, 3D-CTAB reconstruction prevented interference to images of lung tissue and blood parenchyma, and allowed a 360-degree view of the artery branching without blind spots.

In the present study, according to the number of lingual artery, it can be divided into 3 types. We found 3 lingular arteries was in $8.43 \%$ of cases, which was not found by Cory and Valentine, Fourdrain et al., and Dejima et al. (13-15). The interlobar upper division arteries and interlobar lingular arteries were common trunk in $10.89 \%$ of cases, which was significantly lower than that reported by Cory and Valentine $(17.8 \%)$ and Fourdrain et al. $(20.5 \%)(13,14)$. Our study differed from previous studies in the number of lingual artery. The possible reason was that in this study, we used thin-section enhanced CT, coupled with advanced $3 \mathrm{D}$ reconstruction technology, which made the data more accurate and closer to the actual anatomical situation. Therefore, when we performed lingular segmental resection, it was important that attention was paid to the fact that a few patients have multiple lingular segmental arteries, which need to be removed individually to prevent bleeding or incomplete pulmonary segmental resection.

We found that the lingular artery branching patterns also had the following branching types: interlobar (71.68\%), interlobar and mediastinal $(21.08 \%)$, and mediastinal (7.83\%). Jiang reported $76 \%, 18 \%$, and $6 \%$, respectively, whereas Yamashita reported $72.90 \%, 17.6 \%$, and $9.7 \%$, respectively $(8,16)$. Our study is similar to previous studies in the lingular artery branching patterns.

In the present study, the mediastinal lingular artery was seen in 48 cases $(28.91 \%)$, and A4 was the most common type $(12.05 \%)$. In their studies, Boyden and Hartmann and Jiang reported $30 \%$ and $24 \%$ incidence, respectively, and A4b was the most common type (14\% vs. 12\%, respectively) $(7,8)$. However, Hiroaki Nomori reported that A4+5 was the most common type (18\%) (12). Boyden and Hartmann also reported an incidence of $2 \%$ for the mediastinal lingular artery $(\mathrm{A} 4+5)(7)$, but our study did not find an anatomic variation.

The interlobar lingular artery was observed in 119 cases (71.68\%) in the present study; $\mathrm{A} 4 \mathrm{a}, \mathrm{A} 4 \mathrm{~b}$, and $\mathrm{A} 5$ in $7.23 \%$ cases; $\mathrm{A} 4 \mathrm{a}$ and $\mathrm{A} 4 \mathrm{~b}+5$ in $3.01 \%$ cases, and $\mathrm{A} 4 \mathrm{~b}$ and $\mathrm{A} 4 \mathrm{a}+5$ in $4.82 \%$ cases. These anatomical variations have not been reported previously, but the situation was common in the present study. Therefore, care needed to be taken when performing lingual pulmonary segmentectomy. For example, when we performed S5 segmentectomy, A4a or $\mathrm{A} 4 \mathrm{a}$, which is co-stem with $\mathrm{A} 5$, need to be protected.
Moreover, the most common anatomic variant of the interlobar lingular artery was A4+5 (70-80\%) in previously published studies, which was similar to the findings of the present study $(7,8,12)$.

In anatomical segmental pulmonary resection, it is important to understand the branching types of pulmonary segmental and subsegmental arteries. Anatomical variations of the lingual artery are diverse. If the patient has a mediastinal lingular artery, but no preoperative $3 \mathrm{D}$ reconstruction is performed, the mediastinal lingular artery is highly likely to be damaged in the anterior segmental resection of the LUL.

We also observed that in $8.43 \%$ of cases, the origin of A5 was close to A8 or A8+9. Besides in $24.10 \%$ of cases, A5 came from A8 or A8+9, which was seen significantly more than in the previous reports (Jiang 4\%, Yamashita 4.2\%, and Maciejewski and Kutnik 1\%) (8,16,25). However, the findings of the present study were similar to those of Murota et al. (29.7\%) (26).

Inaddition A common trunk for the interlobar upper division artery and interlobar lingular arteries was observed in $10.89 \%$ of the cases. A3a originating from lingular artery accounted for $5 \%$ of cases and A $1+2 \mathrm{c}$ accounted for $3.06 \%$. Our study found that the frequency of this type was lower than previously reported. Boyden and Hartmann reported that $\mathrm{A} 3 \mathrm{a}$ originating from the lingular artery accounted for $13 \%$ of cases, Jiang reported 18\%, Maciejewski and Kutnik reported $16 \%$, and Murota reported $8.1 \%(7,8,25,26)$. Only Murota reported $\mathrm{A} 1+2 \mathrm{c}$ originating from lingular artery accounted for $3.8 \%$ (26). If $3 \mathrm{D}$ reconstruction was not performed before segmentectomy or subsegmentectomy, the $\mathrm{A} 8, \mathrm{~A} 8+9, \mathrm{~A} 3 \mathrm{a}, \mathrm{A} 1+2 \mathrm{c}$, or other arteries may be accidentally damaged with serious consequences.

Therefore, preoperative $3 \mathrm{D}$ reconstruction to understand the segmental lingular artery branching pattern and its rare branching pattern is necessary to perform an accurate pulmonary segmentectomy and subsegmentectomy.

Our study had some limitations. First, we only used 3D-CTAB data. Therefore, it is possible that small arteries may have been missed, which may have biased our results from real anatomical findings. Second, the sample size of the present study was small. A large sample study is needed to confirm the results.

\section{Conclusions}

We identified various lingular artery branching patterns of the LUL with $3 \mathrm{D}-\mathrm{CTAB}$ in patients. 2D-CTAB is useful for 
finding variations. We believe that the findings of the present study can be useful for thoracic surgeons when undertaking segmental and subsegmental pulmonary resections.

\section{Acknowledgments}

Funding: The study was supported by the Wu Jieping Medical Foundation-Clinical Scientific Research Project, China, No. 320.2730.1881; Medical Innovative Project of Fujian Province, China, No2018-CXB-3.

\section{Footnote}

Reporting Checklist: The authors have completed the STROBE reporting checklist. Available at https://dx.doi. org/10.21037/jtd-21-1141

Data Sharing Statement: Available at https://dx.doi. org/10.21037/jtd-21-1141

Conflicts of Interest: All authors have completed the ICMJE uniform disclosure form (available at https://dx.doi. org/10.21037/jtd-21-1141). The authors have no conflicts of interest to declare.

Ethical Statement: The authors are accountable for all aspects of the work in ensuring that questions related to the accuracy or integrity of any part of the work are appropriately investigated and resolved. The study was conducted in accordance with the Declaration of Helsinki (as revised in 2013). The study was approved by ethics board of Fujian Medical University Cancer Hospital, Fujian Cancer Hospital. (NO.: 2017-050-01) and individual consent for this retrospective analysis was waived.

Open Access Statement: This is an Open Access article distributed in accordance with the Creative Commons Attribution-NonCommercial-NoDerivs 4.0 International License (CC BY-NC-ND 4.0), which permits the noncommercial replication and distribution of the article with the strict proviso that no changes or edits are made and the original work is properly cited (including links to both the formal publication through the relevant DOI and the license). See: https://creativecommons.org/licenses/by-nc-nd/4.0/.

\section{References}

1. Altorki NK, Wang X, Wigle D, et al. Perioperative mortality and morbidity after sublobar versus lobar resection for early-stage non-small-cell lung cancer: post-hoc analysis of an international, randomised, phase 3 trial (CALGB/Alliance 140503). Lancet Respir Med 2018;6:915-24.

2. Kamigaichi A, Tsutani $Y$, Fujiwara M, et al. Postoperative Recurrence and Survival After Segmentectomy for Clinical Stage 0 or IA Lung Cancer. Clin Lung Cancer 2019;20:397-403.e1.

3. Keenan RJ, Landreneau RJ, Maley RH Jr, et al. Segmental resection spares pulmonary function in patients with stage I lung cancer. Ann Thorac Surg 2004;78:228-33; discussion 228-33.

4. Kodama K, Higashiyama M, Okami J, et al. Oncologic Outcomes of Segmentectomy Versus Lobectomy for Clinical T1a N0 M0 Non-Small Cell Lung Cancer. Ann Thorac Surg 2016;101:504-11.

5. Suzuki K, Saji H, Aokage K, et al. Comparison of pulmonary segmentectomy and lobectomy: Safety results of a randomized trial. J Thorac Cardiovasc Surg 2019;158:895-907.

6. Suzuki K, Watanabe SI, Wakabayashi M, et al. A singlearm study of sublobar resection for ground-glass opacity dominant peripheral lung cancer. J Thorac Cardiovasc Surg 2020. [Epub ahead of print]. doi: 10.1016/ j.jtcvs.2020.09.146.

7. Boyden EA, Hartmann JF. An analysis of variations in the bronchopulmonary segments of the left upper lobes of fifty lungs. Am J Anat 1946;79:321-60.

8. Ramanathan K, Jia D, Tan CS, et al. Autopsy examination after extracorporeal membrane oxygenation: 'Mortuis vivos docent'. J Thorac Dis 2020;12:1121-3.

9. Sugimoto S, Izumiyama O, Yamashita A, et al. Anatomy of inferior pulmonary vein should be clarified in lower lobectomy. Ann Thorac Surg 1998;66:1799-800.

10. Hagiwara $M$, Shimada $Y$, Kato $Y$, et al. High-quality 3-dimensional image simulation for pulmonary lobectomy and segmentectomy: results of preoperative assessment of pulmonary vessels and short-term surgical outcomes in consecutive patients undergoing video-assisted thoracic surgery dagger. Eur J Cardiothorac Surg 2014;46:e120-6.

11. Ikeda N, Yoshimura A, Hagiwara M, et al. Three dimensional computed tomography lung modeling is useful in simulation and navigation of lung cancer surgery. Ann Thorac Cardiovasc Surg 2013;19:1-5.

12. Nomori H, Okada M. Illustrated Anatomical Segmentectomy for Lung Cancer. Tokyo, Springer; 2012.

13. Cory RA, Valentine EJ. Varying patterns of the lobar 
branches of the pulmonary artery. A study of 524 lungs and lobes seen at operation of 426 patients. Thorax 1959;14:267-80.

14. Fourdrain A, De Dominicis F, Blanchard C, et al. Threedimensional CT angiography of anatomic variations in the pulmonary arterial tree. Surg Radiol Anat 2018;40:45-53.

15. Dejima H, Takahashi Y, Hato T, et al. Mediastinal pulmonary artery is associated with greater artery diameter and lingular division volume. Sci Rep 2017;7:1273.

16. Sato M, Murayama T, Nakajima J. Concepts and techniques: how to determine and identify the appropriate target segment in anatomical pulmonary segmentectomy? J Thorac Dis 2019;11:972-986.

17. Nagata T, Nakamura Y, Kariatsumari K, et al. Potentially overlooked branches of the left pulmonary artery. Asian Cardiovasc Thorac Ann 2016;24:676-80.

18. Mondello B, Lentini S, Barone M, et al. Surgical management of pulmonary inflammatory pseudotumors: a single center experience. J Cardiothorac Surg 2011;6:18.

19. Higashiyama M, Tokunaga T, Nakagiri T, et al. Pulmonary metastasectomy: outcomes and issues according to the type of surgical resection. Gen Thorac Cardiovasc Surg 2015;63:320-30.

20. Lo Faso F, Solaini L, Lembo R, et al. Thoracoscopic lung metastasectomies: a 10-year, single-center experience. Surg
Endosc 2013;27:1938-44.

21. Ichinose J, Kohno T, Fujimori S. Video-assisted thoracic surgery for pulmonary aspergilloma. Interact Cardiovasc Thorac Surg 2010;10:927-30.

22. Nakazawa S, Shimizu K, Mogi A, et al. VATS segmentectomy: past, present, and future. Gen Thorac Cardiovasc Surg 2018;66:81-90.

23. Motono N, Iwai S, Funasaki A, et al. Low-dose indocyanine green fluorescence-navigated segmentectomy: prospective analysis of 20 cases and review of previous reports. J Thorac Dis. 2019 Mar;11:702-7.

24. Subotich D, Mandarich D, Milisavljevich M, et al. Variations of pulmonary vessels: some practical implications for lung resections. Clin Anat 2009;22:698-705.

25. Maciejewski R, Kutnik B. Branches of the left pulmonary artery vascularizing the left upper pulmonary lobe. Acta Anat (Basel) 1990;138:224-9.

26. Murota M, Yamamoto Y, Satoh K, et al. An analysis of anatomical variations of the left pulmonary artery of the interlobar portion for lung resection by three-dimensional CT pulmonary angiography and thin-section images. Jpn J Radiol 2020;38:1158-68.

(English Language Editor: R. Scott)
Cite this article as: He H, Chen P, Chen X, Wang PY, Liu SY, Wang F. Analysis of anatomical variations of the lingular artery of the left upper lobe using 3D computed tomography angiography and bronchography. J Thorac Dis 2021;13(8):50355041. doi: $10.21037 /$ jtd-21-1141 